\title{
AUTONOMY VERSUS EFFICIENCY IN MANAGEMENT OF LARGE-SCALE LOGISTICS NETWORKS
}

\author{
Maurizio Bielli ${ }^{(1)}$, Mariagrazia Mecoli ${ }^{(1)}$ and Agostino Villa ${ }^{(2)}$ \\ (1) Istituto di Analisi dei Sistemi ed Informatica "Antonio Ruberti" \\ Consiglio Nazionale delle Ricerche - Viale Manzoni 30, 00185 Rome, Italy \\ Tel.+3906 77161,Fax+3906 7716461,e-mail : bielli@iasi.cnr.it \\ (2) Dipartimento di Sistemi di Produzione ed Economia dell'Azienda \\ Politecnico di Torino - Viale Duca degli Abruzzi - Torino, Italy \\ Tel.+39011 5647233, e-mail : agostino.villa@polito.it
}

\begin{abstract}
Present evolution of logistics networks is characterized by a progressive increase of the network complexity and the opportunity of organizing management structures composed by local decision-makers (usually denoted "agents"). As much the system complexity and the number of intelligent agents grow, as much the system response uncertainty seems to grow also: this suggests to have greater attention to the agents autonomy and responsibility. Present paper first aims to verify if the increasing complexity of actual logistics systems is going to allow a greater and greater autonomy to individual agents. Copyright ${ }^{\circ} 2005$ IFAC
\end{abstract}

Keywords: Management, Efficiency, Agents, Decentralized systems, Networks

\section{INTRODUCTION}

The globalization of business and production around the world is going to generate a revolution in the logistics systems design and management. The real innovation is evolving along two main lines: on one hand, logistics and production are becoming tasks complementary but performed by different and separated companies; on the other, while production of final goods is going to be performed by groups of firms integrated into "network of enterprises" (Lucertini et al, 1995; Villa, 1998), both internal logistics and distribution is going to be performed by large-scale world-wide service enterprises (Bielli and Villa, 1997). As much the dimension of a logistics network increases, as much the interest of large service network management toward a distributed management organization also increases. The main motivation comes from day-by-day problems in managing a complex network by a unique center. Obviously decentralization of management means that some autonomy must be assigned to local managers, as well as some authority: this decision usually implies caution and doubts in any top manager.

In order to design distributed decision-making architectures, the approach of agent-based technologies and systems was developed as a potential solution to manage and coordinate the complexity of supply chain and logistic networks (Satapathy et al., 1998). In fact, the innovation in the logistics and distribution systems design reflects the general tendency of increasing interest towards cooperative Multi-Agent Systems (MAS) within large-scale distributed organizations. MAS are systems consisting of a number of agents that act with a given degree of autonomy and are able to interact with one another. Each autonomous agent operates in a dynamic environment without any 
external intervention and has some kind of control over its actions and internal state. Agents must realize a set of goals or tasks in order to maximize local profit or efficiency (they are able to map their own inputs to output to maximize their utility) and the decisions they take have to be coordinated and directed towards the global goal of the system. The characteristics of intelligent and autonomous agents have been widely recognized and discussed (Jennings et al., 1998) and their specific applications to the intelligent manufacturing field have been presented and illustrated (Shen and Norrie, 1999).

In recent years many logistics multi-agent based models have been proposed (e.g. within E-commerce Santos et al., 2003, Davidsson, 2004 - 2005, Persson 2005, Timm, et al., 2002), many of them refer to inter-organizational logistics, which emphasize maximizing individual profits. While, in the case where all the agents have a common goal of maximizing the profits of the entire system, as they belong to an unique company, such models address the intra-organizational logistics in order to coordinate the system. Generally all the approaches are lacking any precise optimization model.

The system we refer to is a production and distribution logistics network, where a flow of, say, goods is delivered from producers to customers. Then, logistic networks consist of many loosely connected heterogeneous sets of agents, such as suppliers, firms, companies, retailers, service providers, customers and so on.

The performances of Multi-Agent Systems is defined (see Lee et al.,1998, Dollimore, 1994) as a measure which considers several indicators which include throughput, response time, number of concurrent users, as well as factors introduced by the agent level. However, the relation between local and overall system performance objectives is often rather fuzzy. The aim is thus of designing a model where the local operational performance criteria should be related to the overall level performance goals for the network as a whole. As a consequence, the interest toward a distributed management organization is increasing, where a complex network is managed by a unique center. Therefore, the question is how to derive overall profitability and stability through the distributed individual decisions. In fact, the main resistance against decentralization depends on the doubt about the capacity of a multi-agents organization to assure a high efficiency to the whole system. In practice, in decentralizing management of a large-scale system, as a world-wide logistics network is, top managers ask if when the local autonomy increases, also the global efficiency of the whole system can increase.

To search for a preliminary answer to this question is the goal of the present paper: to verify - if a largescale logistics system, characterized by relevant local responsibility and quality, can generate sufficient space for discretionary decision-making activity of local agents, - and if the consequent local autonomy appears to be as more useful as larger the knowledge uncertainty of all the local operations in the central management is.

Then the paper will approach the following three points:

(1) a decentralized model of a logistics system management suggests that:

a) the global system efficiency can increase if the local agents efficiency increases;

b) the local agents efficiency can increase if a greater and greater local autonomy is allowed; as a consequence,

(2) a model of the design process of a decentralized logistics systems can suggest that the global system efficiency can increase if local agents are characterized by high qualification (i.e. proper responsibility, decision-making capacity, and competence);

(3) a model of the planning process of a decentralized logistics system can suggest that:

a) as higher the local qualification will be, as lower the global efficiency reduction for variations of the operation conditions (e.g. the demands for movements) will be;

b) as more concentrated to specific qualifications the local competence will be, as greater the coordination efforts for limiting the global efficiency reduction for variations in the operation conditions will be also.

\section{A MODEL OF A DECENTRALIZED LOGISTICS SYSTEM MANAGEMENT ORGANIZATION}

The goal of the following model formulation is to have at disposal a simplified but adequate description of a set of agents, each one associated to a proper local agent of a given large-scale logistics system. As a consequence the model to be developed will be oriented:

(a) to describe the global logistics system such as a network of local services, each one devoted to receive and distribute loads according to specific prescriptions of clients (specifying the real demands of movements); to each local service a proper agent is assigned with individual autonomy in managing the local flows addressing;

(b) to represent the throughput flow from each local service such as a function of the utilization rates of the transportation means and capacities of center itself; the utilization rate will specify the control variable at disposal of the local manager, whilst the throughput flow will denote the variable describing interaction of the local center with the other ones in the global logistics system;

(c) to define a significant performance index by which the center manager must estimate the local 
agents efficiency.

The model statement is based on the following notations:

$X_{i} \quad$ net utilization rates, i.e. the local service rates at center $i$;

$W_{i} \quad$ local input flows, i.e. arriving to the center $i$ for being addressed forward;

$Z_{i} \quad$ local throughput flows, i.e. the addressed rates of parts from center $i$ is downstream;

$P_{i}$ (.) local service capacities of center $i$; it proportionally depends on the utilization rate, and is conditioned on the supply of items from upstream;

$S_{i j}$.) transfer rate of items outgoing from center $j$ and addressed to center $i$, depending on client demands;

$U_{i}() ;$.$U performance index (efficiency) of center i$ and of the whole work system, respectively;

$c_{i} \quad$ unitary cost to measure the local efficiency at center $i$, depending on the net utilization rate at the same center;

$L, \mu_{i}, \lambda_{i}$ respectively denote the Lagrangian cost and variables to be used for the mathematical statement of the decision-making problem in terms of mathematical optimization problem, as in the following.

The local throughput flow can be represented as an implicit function of the net utilization rate and of the parts flows arriving to the center $i$ under consideration (see equation (1)). Note that this is a simplified model which approximately shows that, in case of normal operation situation, a local manager of a service center belonging to a large-scale logistics network only needs clear information about the performance of its own center in terms of capacity to address "rapidly and precisely" incoming flows towards downstream centers: this information is sufficient for taking decisions about service rates to be planned.

$$
Z_{i}=P_{i}\left(X_{i}, W_{i}\right)
$$

In the same way the input flow at center $i$ comes from the collection of items flows arriving from upstream centers, according to their respective road map information.

$$
W_{i}=\sum_{j=1}^{N} S_{i j} Z_{j}
$$

The decision-making activity of the local agent is then described by a proper target, formally stated in terms of a performance index, measuring the efficiency of the addressing service to be locally performed, $U_{i}$. We assume that the global system utilization will result from linear composition of individual centers' utilization.

$$
\begin{aligned}
& U=\sum_{i=1}^{N} U_{i} \\
& U_{i}=c_{i} X_{i}
\end{aligned}
$$

Taking into account this last definition of global system utilization (efficiency), the set of local decision-making problems for $i=1, \ldots, N$, (being $N$ the number of service centers in the global logistics system) formally corresponds to a mathematical optimization problem for a Large Scale System (LSS), as analyzed in (Brandimarte and Villa, 1995 and 1999). Its formulation is obtained by stating the Lagrangian, as follows:

$$
\begin{gathered}
L=\sum_{i=l}^{N} U_{i}+\sum_{i=l}^{N} \mu_{i}\left(P_{i}\left(X_{i}, W_{i}\right)-Z_{i}\right)+ \\
\sum_{i=l}^{N} \lambda_{i}\left(W_{i}-\sum_{j=l}^{N} S_{i j} Z_{j}\right)
\end{gathered}
$$

In mathematical terms, solution of this optimization problem can be derived by solving the well known optimization conditions (see Singh and Titli, 1979). But the problem to be approached now is referred to the conceptual utilization of said model to analyze how global and local efficiency can be affected by the agents' autonomy.

\section{REALISTIC COORDINATION OF LOCAL AUTONOMOUS AGENTS}

The above mathematical formulation can have two types of interpretations:

$>$ on one hand, despite its denomination, it states a "centralized optimization problem", that means the problem of optimizing a LSS through a decomposition based on the mathematical structure of the constraints;

$>$ on the other, it allows to derive a "deterministic optimization task", that means a procedure for computing an optimized strategy for deterministic production planning (i.e., planning in a completely and perfectly known situation).

A question immediately arises: where are the agents? Which parts of the decision-making model may describe a local autonomous decision?

To answer this question, a different interpretation of the outlined model, as well as of its utilization for management purpose, must be adopted. Let us consider the description of a decentralized subproblem $i$, as stated by conditions (1) and (4), the last one denoting a local performance index to be maximized. A practice-oriented interpretation can state that condition (1) describes impact of the agent's decision on the service rate of center $i$. Then, conditions (1) and (4) specifies a deterministic model of a decision-making activity at local level: they do 
not describe an agent, because they don't include neither express any effect of local autonomy.

An effective improvement of the above stated local model is then to modify it in such a way to describe autonomy, i.e. how the agent can take discretionary decision. To this aim, let us consider the point of view of a real top manager, the responsible of the whole logistics system who is in charge of assuring co-ordination of local decision-makers and then has the authority of assigning them an effective autonomy. The top manager must decentralize autonomy in form of discretionary power, provided this freedom in deciding is assigned to "responsible" agents (i.e., agents who agree in respecting the global system regulations). Moreover, the top manager must realize that he cannot a priori and perfectly know decision which will be taken by each agent. This one will operate after he has received his own mission, and he will try to solve his own local management problem by applying his own knowledge, procedures and criteria. As a consequence the top manager must suppose that he can only have an approximated estimation of what locally will be decided. This approximation affects his a priori knowledge of any local decision-making process and it can be stated as the "knowledge uncertainty" of the top manager about local decisions. A simple model of this uncertainty can be written as:

$$
Z_{i}=P_{i}\left(X_{i}, W_{i}\right)+\xi_{i}
$$

where, for instance, $\xi_{i}=\operatorname{Normal}\left(0, \sigma_{i}\right)$.

When introducing condition (6) instead of (1), the mathematical problem modifies into a stochastic optimization. But the aim here is different from solving this task. Now the goal is to show how the global plant efficiency is affected by local autonomy. And this added condition help us in stating a model of local autonomy: "the approximation of the a priori knowledge of top manager with reference to local agents' decision is a measure of the local agent autonomy".

Then, the above introduced model (6) of the uncertainty associated to local decision-making activity is also a model of the local autonomous decision-making process, as it is understood by the central co-ordination manager.

The modified model of the local agents will allow to derive some considerations concerning potential relations between local autonomy and global efficiency, just making reference to well known results of LSS theory. A first peculiarity of the global problem solution comes from noting the relation between system complexity (i.e. the number of component centers) and uncertainty of the overall system response (then, uncertainty of top manager in dealing with the local agents co-ordination).
Result 1: Uncertainty in the local agents coordination proportionally depends on the overall system complexity.

This result directly derives from stochastic optimization theory, when the overall problem (1)-(4) is considered such as a standard unique optimization problem in which constraints are affected by additive noise. A complementary peculiarity of the global problem solution can be proven by noting the relation between the overall system efficiency $U$ and the coordination action (as outlined by Lagrangian variables in $(5))$.

Result 2: The overall system efficiency proportionally depends on the effectiveness of the co-ordination.

This second result is just a rewriting of an intrinsic peculiarity of the Lagrangian decomposition approach to the LSS decentralized optimization. In the present application frame, it suggests clear actions to the top management, towards local agents. Co-ordination indeed can be affected by local uncertainty but it always plays a benefic role: its strength is more and more useful also in uncertain situations. Again by applying properties of the LSS mathematical optimization theory a further peculiarity of the global problem solution can be proven, now referred to the co-ordination action.

Result 3: An increase of the co-ordination effectiveness is positively related to an increment in the local efficiency.

Proof comes from noting that, in Lagrangean formulation, as better the local efficiency is, as greater the co-ordination action must be in order to assure optimized interactions (Dobson and Karmarkar, 1995). In practice, it means that an efficient management of individual local services requires a better and better co-ordination by top manager: this appears to be the effectively crucial condition for a continuously improved management of a multi-agents system. A final peculiarity can be obtained as direct derivation of the above two results, because they allow to link together global and local efficiencies.

Result 4: In case Result 3 is assured, then the overall system efficiency positively depends on the local efficiency.

Proof of this last result comes from "serialization" of the statements of the above Results 2 and 3. A more interesting version of Result 4 comes from noting that the "local efficiency" of every agent is directly related to the agent's autonomy, as we have discussed in previous Sections. As it has been observed several times, autonomy pushes agent to be motivated in his own decision-making activity, then it forces agent to be efficient.

Then, Result 4 can be rewritten as: "The overall production system efficiency as much increases as much autonomy of local agents is guaranteed".

A more detailed investigation of these preliminary results with an application to the case of workflow 
management systems in extended enterprises has been presented (Villa, 2002). In particular, the problems of decentralized production management organization and coordination / cooperation of local autonomous agents are discussed by analysing the relationships among overall efficiency, effectiveness of the coordination, autonomy and efficiency of local cooperative agents.

\section{CONCLUSIONS}

Considerations derived in the above Section clearly summarizes the main result of this paper:

"In a large-scale decentralized logistics system composed by service centers able to locally address flows and individually managed by autonomous agents, the global efficiency can be improved as much as the local autonomy is assured".

As remarked in LSS theory, in any large-scale system the best possible performance of the whole system can be obtained by means of decentralized optimization procedures. In case of local logistics service centers controlled by intelligent agents, the mathematical concepts of "LSS decentralization" is directly related to the organizational concept of "agent autonomy", that means local opportunity for discretionary decision (Jones and Jasek, 1998; Krothapalli and Deshmuhk, 1999).

It must be noted, however, that what has been here presented is preliminary only, because a formal proof of the presented four Results must still be completed.

What can be already remarked is the new utilization of the formal models of local agents for representing the concept of "local autonomy". This concept deserves two complementary interpretations: on one hand, local autonomy reflects into central uncertainty of the top manager about the effects of local decisions; on the other, local autonomy is the real key for allowing effective decision-making alternatives to local agents, and then assuring them an increased efficiency. And an increased local efficiency reflects into a potentially more effective integration of any local agent into the global management organization, provided the agent be responsible. This chain of considerations still need to receive better formulation: anyway they just now offer to manager of world-wide logistics networks preliminary tools for improving their networks' design and management.

Acknowledgement: This work has been partially supported by the Italian Consiglio Nazionale delle Ricerche (C.N.R.), Rome.

\section{REFERENCES}

Bielli M., and Villa A. (1997). Research perspectives in industrial logistics design, in Designing Innovations in Industrial Logistics Modelling (Bielli M. and Kusiak A., Eds.), pp. 3-19, CRC Press, Boca Raton, CA.

Brandimarte, P., and Villa, A. (1995). Advanced Models for Manufacturing Systems Management, CRC Press, Boca Raton.

Brandimarte, P., and Villa, A. (Eds.), (1999). Modeling Manufacturing Systems: From Aggregate Planning to Real-Time Control, Springer-Verlag, Berlin.

Davidsson, P., Henesey, L.. Ramstedt, L. Törnquist, J., Wernstedt, F. (2005), Agent-Based Approaches to Transport Logistics, to appear in the Whitestein Agent Technology Series.

Davidsson, P. and Wernstedt, F. (2004), A Framework for Evaluation of Multi-Agent System Approaches to Logistics Network Management, Multi-Agent Systems: An Application Science, Kluwer.

Dobson, G., and Karmarkar, U.S. (1995). Large-scale job scheduling by Lagrangian decomposition, in Optimization Models and Concepts in Production Management, (P. Brandimarte and A. Villa Eds.), pp. 45-70, Gordon \& Breach, Amsterdam.

Dollimore, J. and Kindberg T., (1994). Distributed systems: concepts and design, Second edition, George Coulouris, Addison-Wesley.

Jennings, N. R., Sycara, K., Wooldridge, M. (1998). A Roadmap of Agent Research and Development, Autonomous Agents and MultiAgent Systems, 1, pp. 7-38.

Jones, P., and Jasek, C.A. (1998). From distributed supervisory control to enterprise integration: coordination support for operational environment, Group Decision and Negotiation, 7, pp. 249-264.

Krothapalli, N.K., and Deshmukh, A.V. (1999). Design of negotiation protocols for multiagent manufacturing systems, International Journal of Production Research, 37, pp.16011624.

Lee, L. et al., (1998). The stability, scalability and performance of multi-agents systems, BT Technology Journal, 16, 3, pp.94-103.

Lucertini, M., Nicolò, F., Rossetto, S., Telmon, D., Ukovich, W. and Villa, A. (1995). How to evaluate integration between operations management and organizational structures in CIM design, Control Engineering Practice, A Journal of IFAC, 3, pp.51-58

Persson, J. and Davidsson, P., (2005), Integrated Optimization and Multi-agent Technology for Combined Production and Transportation Planning, HICSS-38.

Santos, E. and Zhang F. (2003). Intra-organizational logistics management through multi-agents systems, Electronic Commerce Research, 3, pp. 337-364. 
Satapathy, G., Kumura, S. R. T., Moore, L. M., (1998). Distributed intelligent architecture for logistics (DIAL), Expert Systems With Applications, 14, pp. 409-424

Shen, W., Norrie, D. H., (1999). Agent -Based Systems for Intelligent Manufacturing: A State-of-the-Art Survey, Knowledge and Information Systems, an International Journal, 1(2), pp. 129-156.

Singh, M.G. and Titli A. (1979). Handbook of large scale systems engineering and applications, North Holland, Amsterdam.
Timm, I. J. et al. Eds. (2002). Agent technologies in logistics, Proceedings of the ECAI-02 Workshop, July 23, Lyon, France.

Villa, A. (1998). Organizing a "network of enterprises": an object-oriented design methodology, Computer Integrated Manufacturing Systems, 11, pp. 331-336.

Villa, A. (2002). Autonomy versus efficiency in multi-agents management of extended enterprices, Journal of Intelligent Manufacturing, 13, pp. $429-438$. 